Bundesgesundheitsbl 2014 · 57:998-1001 DOI 10.1007/s00103-014-2005-5

๑) Springer-Verlag Berlin Heidelberg 2014

Bekanntmachung des Umweltbundesamtes

\title{
Beurteilung materialbürtiger Kontaminationen des Trinkwassers
}

\author{
Empfehlung des Umweltbundesamtes \\ (UBA) nach Anhörung der Trinkwasser- \\ kommission des Bundesministeriums für \\ Gesundheit (BMG) beim Umweltbundesamt
}

\section{Zweck der Empfehlung}

\section{Zusammenfassung: Anwendbarkeit von Leitwerten zur Beurteilung materialbürtiger Stoffe im Trinkwasser}

Wenn das Trinkwasser durch einen materialbürtigen Stoff verunreinigt ist, für den die Trinkwasserverordnung keinen Grenzwert enthält, und das Gesundheitsamt - etwa im Rahmen von $\$ 9$ Absatz 7 oder $\$ 20$ Absatz 1 TrinkwV 2001- zu bewerten hat, ob die Verunreinigung eine Schädigung der menschlichen Gesundheit nach $₫ 6$ Absatz 1 TrinkwV 2001 besorgen lässt, dann kann es hierfür die Leitwerte für materialbürtige Kontaminanten nach den Leitlinien und Bewertungsgrundlagen des Umweltbundesamtes (UBA) heranziehen. Die Leitwerte für materialbürtige Kontaminanten [in den UBA-Leitlinien zur hygienischen Beurteilung von organischen Materialien im Kontakt mit Trinkwasser bisher als DWPLL (Drinking Water Positive List Limit)-Werte bezeichnet] sind humantoxikologisch abgeleitete provisorische Trinkwasserhöchstwerte für materialbürtige Stoffe. In den Leitlinien des Umweltbundesamtes zur hygienischen Beurteilung von organischen $\mathrm{Ma}$ terialien im Kontakt mit Trinkwasser dienen sie zur Definition einer im Prüfsystem als akzeptabel zu bewertenden Stoffmigration zu dem in der Leitlinie festgelegten Zeitpunkt.
Die Empfehlung unterstützt die Gesundheitsämter bei der gesundheitlichen Bewertung von Kontaminationen des Trinkwassers, die durch Werkstoffe und Materialien, die in Wasserversorgungsanlagen verbaut werden, verursacht worden sein könnten.

Zur Bewertung der Analyseergebnisse bei Kontaminationen, die auf eine Verunreinigung des Rohwassers (z. B. durch Emissionen aus Altablagerungen) zurückgehen, ist die Empfehlung nicht vorgesehen.

\section{Anzeichen für materialbürtige Kontaminationen des Trinkwassers}

\subsection{Anzeichen für metallene Verunreinigungen}

Verunreinigungen des Trinkwassers, die durch metallene Werkstoffe verursacht sind, können teilweise anhand von Trübung und Färbung (z. B. grün/blau durch Kupferverbindungen oder braun durch Rost aus Eisen) erkannt werden, und die Endabnehmer des Wassers beanstanden dies in der Regel auch.

Die häufigsten Kontaminanten, verursacht durch metallene Werkstoffe, sind Eisen, Kupfer, Blei und Nickel, die alle per
Grenzwert in der Trinkwasserverordnung geregelt sind und deren Konzentrationen einer entsprechenden Untersuchung des Trinkwassers auch entsprechend bestimmt werden.

\subsection{Anzeichen für organische Verunreinigungen}

Bei routinemäßigen oder umfassenden Trinkwasseruntersuchungen werden Verunreinigungen des Trinkwassers durch Stoffe aus organischen Materialien häufig nicht erfasst, denn die Trinkwasserverordnung enthält nur wenige entsprechende Parameter. Eher werden materialbürtige organische Kontaminationen bei der Verwendung des Trinkwassers wahrgenommen. Insbesondere fallen plötzliche Beeinträchtigungen der Trinkwasserqualität durch einen fremdartigen Geruch oder Geschmack auf, z. B. während oder nach baulichen Maßnahmen an der Trinkwasser-Installation. Dies verunsichert die Endabnehmer entsprechend und lässt sie gesundheitliche Beeinträchtigungen befürchten.

Auch Sanierungsmaßnahmen der Trinkwasser-Installation (z. B. Beschichtungen zur Rohrinnensanierung) können $\mathrm{zu}$ solchen Befürchtungen führen, insbesondere wenn dabei nicht zertifizierte Verfahren zum Einsatz kommen. 


\section{Ursachen materialbürtiger Kontaminationen}

Materialbürtige Kontaminanten stammen selten aus dem Verteilungsnetz des Wasserversorgers. Wahrscheinlichere Quelle ist die Trinkwasser-Installation von Wohnhäusern mit ihren viel höheren (ungünstigeren) Oberflächen/Volumen-Verhältnissen der Rohrleitungen, oft höheren Wassertemperaturen, längeren Stagnationszeiten und vielfältigeren Materialien.

\subsection{Ursachen metallener Kontaminationen}

Als metallene Verunreinigungen des Trinkwassers treten hauptsächlich Blei, Kupfer, Nickel und Eisen auf. Blei kann aus noch vorhandenen Bleileitungen ins Trinkwasser gelangen. Aber auch bleihaltige Kupferlegierungen können Blei ins Trinkwasser abgeben. Erhöhte Kupferkonzentrationen sind in der Regel durch blanke Kupferrohre verursacht, wenn die Voraussetzungen für die Verwendung von Kupferrohren (s. DIN 50930-6) nicht erfüllt sind. Nickel wird vor allem von verchromten oder vernickelten Bauteilen ins Trinkwasser abgegeben. Erhöhte Eisenkonzentrationen werden entweder durch verzinkte Stahlrohre verursacht, deren Zinkschicht (teilweise) verschwunden ist, oder durch gusseiserne Rohre des Wasserversorgers, in denen das Wasser zeitweise stagniert.

\subsection{Ursachen organischer Kontaminationen}

In Wasserversorgungsanlagen werden immer häufiger vielfältige organische Materialien verbaut. Den Hauptanteil stellen die Kunststoffmaterialien für Rohrleitungen. Darüber hinaus sind Elastomermaterialien (Gummi, Silikone, thermoplastische Elastomere) für Dichtungen und Schläuche unverzichtbar und kommen in Trinkwasser-Installationen besonders häufig vor. Daneben finden andere Materialien wie Epoxidharze, Polyurethane, Polyacrylate und Polyester in Form von Beschichtungen oder als Klebstoffe und Dichtungsmittel in Behältern, Rohrleitungen oder kleinen Bauteilen für Trinkwasser-Installationen Verwendung. Auch Sa- nitärarmaturen, Fittinge, Absperrschieber und andere sog. Ausrüstungsgegenstände für Wasserversorgungsanlagen enthalten organische Materialien in unterschiedlichen Anteilen.

Organische Materialien bestehen aus Polymermolekülen, die sich wegen ihrer Größe und oft hydrophoben Eigenschaften nicht im Wasser lösen. Polymermaterialien können jedoch Restmonomere und andere Ausgangsstoffe enthalten, die in das Trinkwasser übergehen können. Insbesondere werden den Polymeren Additive zugesetzt, die die technische Eigenschaften, wie Verarbeitbarkeit oder Langzeitstabilität, gewährleisten sollen. Die wichtigsten und am häufigsten eingesetzten Additive sind Stabilisatoren gegen thermischen oder durch UVLicht induzierten Molekülkettenabbau. Die Stabilisatoren werden im Laufe des „Lebens“ eines Kunststoffproduktes verbraucht, indem sie sich zersetzen und dadurch die Polymerketten stabilisieren. Dabei entstehen auch Zersetzungsprodukte, die als freie Moleküle im Polymer verbleiben. Manche Zersetzungsprodukte sind ausreichend wasserlöslich und können in das Trinkwasser übergehen.

Polyzyklische aromatische Kohlenwasserstoffe (PAK) oder Benzo(a)pyren (BaP) sind Parameter der Trinkwasserverordnung. Diese Stoffe können Kontaminanten des Rohwassers sein, sie können aber auch aus sehr alten Wasserleitungen aus Stahl, Gusseisen oder schwarzem Eisen, die früher mit Teer oder Bitumen unklarer Herkunft oder Qualität korrosionsgeschützt wurden, ins Trinkwasser abgegeben werden. Im Verteilungsnetz der Wasserversorger werden derartige Rohrleitungen seit Jahrzehnten nach und nach durch einwandfreie Leitungen ersetzt. Aber auch in Kleinanlagen zur Eigenversorgung (nach $₫ 3 \mathrm{Nr}$. 2 Buchstabe c Trinkwasserverordnung) können derart beschichtete Rohre noch vorhanden sein.

Aufgrund der großen Vielfalt der möglicherweise in Bauteilen aus Kunststoff verwendeten Ausgangsstoffe und der häufig fehlenden Dokumentation über die in der Installation verwendeten Bauteile ist es in der Regel schwierig, beim Auftreten einer Beeinträchtigung der Trinkwasserqualität, z. B. durch auffälligen Geruch oder Geschmack, gezielt diejenigen Stoffe anzugeben, auf die das Trinkwasser untersucht werden sollte.

Geruch und Geschmack des Trinkwassers können auch dadurch beeinträchtigt sein, dass das eventuell vorhandene Desinfektionsmittel Chlor mit Migrationsstoffen reagiert hat. Auch in diesem Fall ist es schwierig, konkrete Stoffe zu benennen, die im Wasser untersucht werden sollten, um die Ursache der organoleptischen Veränderung zu bestimmen.

\section{Anforderungen an Materialien und Werkstoffe im Kontakt mit Trinkwasser}

\subsection{Leitlinien/Bewertungsgrundla- gen des Umweltbundesamtes}

Das Umweltbundesamt veröffentlichte in der Vergangenheit Leitlinien und Empfehlungen ${ }^{1}$, die die allgemeinen Anforderungen an Materialien im Kontakt mit Trinkwasser des $\$ 17$ der Trinkwasserverordnung konkretisierten. Mit der Änderung der Trinkwasserverordnung vom 05.12.2012 erhielt das Umweltbundesamt die Aufgabe, verbindliche Bewertungsgrundlagen für Materialien im Kontakt mit Trinkwasser festzulegen. Die Einhaltung dieser Anforderungen - sowohl der momentan noch gültigen Leitlinien als auch der neuen Bewertungsgrundlagen - kann durch ein Zertifikat eines für den Trinkwasserbereich akkreditierten Zertifizierers bestätigt werden. In diesem Fall ist davon auszugehen, dass die entsprechenden Produkte in Ordnung sind.

\subsection{Toxikologisch begründete Leitwerte in den Leitlinien des UBA}

Zur hygienischen Beurteilung von organischen Materialien im Kontakt mit Trinkwasser verwenden sowohl die Leitlinien als auch die künftigen Bewertungsgrundlagen des UBA toxikologisch begründete Leitwerte, anhand derer die Stoffabgabe der Produkte aus dem jeweiligen Material an das Prüfwasser, in Abhängigkeit von der Rezeptur des Herstellers, zu be-

\footnotetext{
1 http://www.umweltbundesamt.de/wasser/ themen/trinkwasser/verteilung.htm.
} 
urteilen ist. In den Leitlinien heißen diese Leitwerte bisher Drinking Water Positive $L$ ist Limit $=$ DWPLL-Werte. Sie finden sich in den Positivlisten der einzelnen Leitlinien.

Voraussetzung für die Aufnahme eines Stoffes in die Positivliste einer Leitlinie ist seine vollständige humantoxikologische Bewertbarkeit und Bewertung. Die auf letzterer basierenden gesundheitlichen Leitwerte repräsentieren in der Regel $10 \%$ der lebenslang gesundheitlich duldbaren Belastung und stellen dadurch sicher, dass selbst nach lebenslanger täglicher Aufnahme der Substanz bis zur Höhe ihres Leitwertes eine Schädigung der menschlichen Gesundheit nicht zu besorgen wäre.

Für einige Materialien sind in den Leitlinien weitere Leitwerte als „Zusatzanforderungen " festgelegt. Solche Zusatzanforderungen begrenzen Stoffe oder Stoffgruppen, die als Reaktionsnebenprodukte oder Abbauprodukte im organischen Material vorliegen und als solche in das Trinkwasser übergehen können. Die Einhaltung dieser zusätzlichen Parameter ist für die Bestätigung, dass ein Produkt die Anforderungen der Leitlinien bzw. Bewertungsgrundlagen erfüllt, unabhängig von der Rezeptur materialspezifisch (organische Beschichtungen, Elastomere) zu überprüfen.

\subsection{Begründung der Leitwerte in den Leitlinien/ Bewertungsgrundlagen}

Die meisten der in den Leitlinien gelisteten Stoffe wurden vom CEF Panel der europäischen Lebensmittelbehörde EFSA (früher SCF) für Kunststoffe im Lebensmittelkontakt (Verpackungsmaterialien) bewertet. Die Bewertungsvorschläge der EFSA führen zur Aufnahme der bewerteten Stoffe in die Positivliste der Verordnung (EU) Nr. 10/2011. Falls der Stoff eine Migrationsbeschränkung als ,Specific migration limits" (SML) erhält, stellt dieser ein Grenzwerte für materialbürtige Stoffe in Lebensmitteln dar. Dabei gilt die Konvention, dass $1 \mathrm{~kg}$ Lebensmittel in $6 \mathrm{dm}^{2}$ verpackt wird und die tägliche Aufnahme eines so verpackten Lebensmittels maximal $1 \mathrm{~kg}$ beträgt. Für die Ableitung von Trinkwasser-Leitwerten aus den SML-
Werten werden diese durch $20 \mathrm{l} / \mathrm{kg}$ dividiert. Dieser Divisor ergibt sich aus der Annahme, dass eine Person pro Tag nicht mehr als 21 Trinkwasser aufnimmt; mit der darin enthaltenen Konzentration des Stoffes soll sie maximal $10 \%$ (=Allokation) der unbedenklichen Gesamtaufnahme eines Stoffes pro Tag ausschöpfen, da weitere Belastungen mit diesem Stoff aus anderen Quellen (z. B. aus Lebensmitteln) hinzukommen können.

In bestimmten Fällen sind auch kanzerogene oder mutagene Stoffe als Ausgangsstoffe (z. B. Monomere) für die Herstellung von organischen Materialien akzeptiert, wenn diese ein toxikologisches Zulassungsverfahren für Lebensmittelkontaktmaterialien durchlaufen haben. Der Restgehalt im Polymer wurde für kanzerogene Stoffe in Form eines QM-Wertes ${ }^{2}$ festgelegt und soll möglichst niedrig sein, damit diese Stoffe nicht ins Lebensmittel oder in das Trinkwasser migrieren können. Für kanzerogene Stoffe wurde der SML-Wert mit "nicht nachweisbar" festgelegt. Der Trinkwasser-Leitwert für diese Substanzen, wenn für sie keine Grenzwerte der Trinkwasserverordnung (wie für Vinylchlorid, Epichlorhydrin oder Acrylamid) festgelegt sind, richtet sich nach der angenommenen Nachweisgrenze von $0,1 \mu \mathrm{g} / \mathrm{l}$.

\section{Vorgehen zur Bestimmung und Bewertung materialbürtiger Kontaminationen des Trinkwassers}

Bei einem Verdacht einer materialbürtigen Kontamination sollte, soweit möglich, überprüft werden, ob für die verbauten Produkte der Trinkwasserinstallation Bestätigungen der trinkwasserhygienischen Eignung (Zertifikate oder Prüfzeugnisse) vorliegen. Besonders bei älteren Anlagen fehlen häufig entsprechende Dokumente oder überhaupt eine Übersicht der Trinkwasserinstallation mit den darin verbauten Produkten.

\footnotetext{
2 Bezeichnung für den maximalen Restgehalt im Endprodukt (limit on the residual quantity left in the finished material or article).
}

\subsection{Bestimmung und Bewertung metallener Kontaminationen}

Liegt ein Verdachtsfall vor, dass eine materialbürtige metallene Kontamination im Trinkwasser einer bestimmten Entnahmestelle vorliegt (z. B. Blei), kann dieser Verdacht durch eine Probennahme mit einem geeigneten Analysenverfahren überprüft werden. Hierzu sollte eine gestaffelte Probennahme entsprechend der UBA-Empfehlung „Beurteilung der Trinkwasserqualität hinsichtlich der Parameter Blei, Kupfer und Nickel" erfolgen, in der auch die Bewertung beschrieben ist. Die dort beschriebene Probennahme und die Bewertung können auch für andere metallene Verunreinigungen (z. B. Antimon oder Arsen) unter Berücksichtigung der jeweiligen Grenzwerte der Trinkwasserverordnung angewendet werden. Zur Beurteilung von Kontaminationen mit Elementen, die nicht mit einem Grenzwert der Trinkwasserverordnung belegt sind, können - sofern vorhanden - Trinkwasser-Leitwerte der WHO oder Leitwerte des Umweltbundesamtes herangezogen werden.

\subsection{Bestimmung und Bewertung organischer Kontaminationen}

Im Falle einer Beeinträchtigung des Geruchs oder Geschmacks des Trinkwassers, die durch organische Stoffe verursacht ist, sollte auch geprüft werden, ob eine mikrobielle Verunreinigung vorliegt. Diese kann ebenfalls zu olfaktorischen Qualitätsbeeinträchtigungen des Trinkwassers führen. Wie oben bereits erwähnt, wird in der Regel eine Vermutung über einzelne für den festgestellten Geruch oder Geschmack verantwortliche materialbürtige Substanzen kaum möglich sein (da die Rezepturen der verbauten organischen Materialien selten zur Verfügung stehen); eine solche Hypothese wäre aber Voraussetzung für eine gezielte Einzelstoffanalytik. Hinweise auf die Kontaminanten kann ein GC-MS-Screening-Verfahren liefern, mit dem sichergestellt ist, dass ein breites Spektrum der leicht flüchtigen Substanzen (zu denen Geruchs- und Geschmacksstoffe häufig zählen) erfasst wird. Sollten organische Beschichtungen als Ursache der Beeinträchtigung des Trinkwassers vermutet werden, kann das 
Trinkwasser auf die in der Beschichtungsleitlinie aufgeführten Zusatzanforderungen (z. B. primäre aromatische Amine, Bisphenol-A, Bisphenol A/F-diglycidylether und seine Hydrolyse- und Chloraddukte) untersucht werden.

Die Probennahme von Kaltwasser zur Untersuchung von organischen Stoffen sollte - wie die für Metalle - als gestaffelte Stagnationsprobe entsprechend der UBAEmpfehlung „Beurteilung der Trinkwasserqualität hinsichtlich der Parameter Blei, Kupfer und Nickel“ erfolgen. Für die Probennahme sind in diesem Fall jedoch Glasflaschen zu verwenden.

Sollte eine Probennahme für Warmwasser vorgesehen werden, ist hierfür die Art der Warmwasserverteilung zu berücksichtigen. Grundsätzlich ist darauf zu achten, dass die Probennahme die örtlichen technischen Gegebenheiten und Nutzungsbedingungen zutreffend abbildet. Bei einer zentralen Warmwassererzeugung, die mit einer Zirkulationsleitung ausgestattet ist, reicht eine Probennahme aus, nachdem etwas Wasser abgelaufen ist. In anderen Fällen sollte eine für die örtlichen Gegebenheiten spezifische Probennahme durchgeführt werden.

Sollten bestimmte materialbürtige organische Stoffe im Trinkwasser identifiziert werden, für die in den UBA-Leitlinien und in den zukünftigen Bewertungsgrundlagen des UBA Leitwerte für materialbürtige Kontaminanten (bislang „DWPLL-Werte") festgelegt sind, können diese Werte für die gesundheitliche Bewertung der ermittelten Konzentrationen herangezogen werden.

\subsection{Vorgehen bei Neuinstallationen}

Bei Neuinstallationen ist es akzeptabel, dass nicht sofort alle Anforderungen eingehalten werden. Bei metallenen Werkstoffen wird akzeptiert, dass die Ausbildung einer schützenden Deckschicht bis zu 16 Wochen in Anspruch nimmt. Bei organischen Materialien sollten spätestens nach 4 Wochen alle Anforderungen (auch an den Geruchsschwellenwert) eingehalten werden. Bis dahin sollten die Konzentrationen im Trinkwasser den 10-fachen Leitwert der UBA-Leitlinien nicht überschreiten. 